\title{
No evidence for killer sperm or other selective interactions between human spermatozoa in ejaculates of different males in vitro
}

\author{
H. D. M. Moore ${ }^{1,2^{*}}$, M. Martin ${ }^{2}$ and T. R. Birkhead ${ }^{3}$ \\ Departments of ${ }^{1}$ Obstetrics and Gynaecology, ${ }^{2}$ Molecular Biology and Biotechnology and ${ }^{3}$ Animal and Plant Sciences, \\ University of Sheffield, Sheffield S10 2TN, UK
}

\begin{abstract}
This study examines one of the possible mechanisms of sperm competition, i.e. the kamikaze sperm hypothesis. This hypothesis states that sperm from different males interact to incapacitate each other in a variety of ways. We used ejaculates from human donors to compare mixes of semen in vitro from the same or different males. We measured the following parameters: (i) the degree of sperm aggregation, velocity and proportion of morphologically normal sperm after 1 and $3 \mathrm{~h}$ incubation in undiluted semen samples, (ii) the proportion of viable sperm plus the same parameters as in (i) in 'swim-up' sperm suspensions after 1 and $3 \mathrm{~h}$ incubation, (iii) the degree of self and non-self sperm aggregation using fluorescent dyes to distinguish the sperm of different males, and (iv) the extent of sperm capacitation and acrosome-reacted sperm in mixtures of sperm from the same and different males. We observed very few significant changes in sperm aggregation or performance in mixtures of sperm from different males compared with mixtures from the same male and none that were consistent with previously reported findings. The incapacitation of rival sperm therefore seems an unlikely mechanism of sperm competition in humans.
\end{abstract}

Keywords: sperm competition; mechanism of sperm competition; human reproduction; kamikaze sperm hypothesis

\section{INTRODUCTION}

Sperm competition is the competition between the ejaculates of different males for fertilization of a female's ova (Parker 1970). Among species with internal fertilization sperm competition occurs when a female copulates with and is inseminated by two or more males during a single breeding cycle. Throughout a wide range of animal taxa, including reptiles, birds and mammals, sperm competition is widespread and is a powerful selective force shaping the morphology, behaviour and physiology of reproduction (Smith 1984a; Birkhead \& Møller 1998). In most cases females appear to seek multiple copulation partners actively and the types of benefit females might obtain have been extensively discussed (Birkhead \& Parker 1997). The mechanistic aspects of sperm competition have been studied in detail in only a small number of species. In insects and birds, the last male to inseminate a female before ovulation usually fertilizes the majority of ova. Last male sperm precedence, as it is called, occurs through a variety of processes. In insects, incoming spermatozoa may displace those of previous inseminations (e.g. Simmons et al. 1999) or substances in the male's seminal fluid may incapacitate spermatozoa from previous inseminations stored in the female (Harshman \& Prout 1994; Chapman et al. 1995; Clark et al. 1995; Price et al. 1999). In birds, last male sperm precedence arises largely as a consequence of passive sperm loss

*Author for correspondence (h.d.moore@sheffield.ac.uk). between successive inseminations (Birkhead 1998). In mammals with induced ovulation, the first male usually fertilizes the ova but in spontaneous ovulators there are no consistent insemination order effects (Gomendio et al. 1998). Instead, the outcome of inseminations by different males is thought to be determined by interactions between the order of copulation, the interval between copulations and the timing of the insemination relative to when the female ovulates (Ginsberg \& Huck 1989; Huck et al. 1989). In addition to these basic mechanisms in all species, other factors may also influence the outcome of sperm competition. These include differences between males in the rate at which spermatozoa are capacitated (Moore \& Bedford 1983), the velocity of spermatozoa (Holt et al. 1996; Froman \& Feltman 1998), the relative fertilizing capacity of spermatozoa (Dzuik 1996; Birkhead et al. 1999) and the compatibility between spermatozoa and the female tract and ova (Zeh \& Zeh 1997; Clark et al. 1999).

Baker \& Bellis (1988) proposed an additional mechanism of sperm competition in mammals. Their kamikaze sperm hypothesis states that the variation which exists in sperm morphology within an ejaculate is not the result of production errors during spermatogenesis, as other studies have indicated (Cohen 1967, 1973; see also Manning \& Chamberlain 1994), but instead is an adaptation to sperm competition. This idea was previously introduced by Sivinski (1980) and Silberglied et al. (1984) to explain the variation in insect sperm. Specifically, Baker \& Bellis (1988) proposed that, within 
an ejaculate, there are two broad categories of spermatozoa. So-called 'egg getters' were spermatozoa which were capable of fertilizing the oocyte and 'kamikaze' spermatozoa were those which would sacrifice themselves in the 'warfare' between spermatozoa of different males when a female is inseminated by two or more males. Baker \& Bellis (1988) suggested that only a small proportion of spermatozoa (with large-headed morphology) were capable of fertilization and that the role of the other spermatozoa was 'warfare'. They asserted that certain spermatozoa with oval-headed morphology had evolved to kill or incapacitate the spermatozoa of rival males (Baker \& Bellis 1988, 1989). Using data from humans, Baker \& Bellis (1995, p. 274) subsequently reported that, when they mixed semen from two different males in vitro, they observed the following statistically significant changes: (i) a reduction in the proportion of oval-headed sperm (otherwise known as morphologically normal sperm; World Health Organization 1992), (ii) an increase in agglutination, (iii) an increase in mortality, and (iv) a reduction in sperm velocity compared with controls in which self-spermatozoa had been mixed. Their explanation for these effects was that, on making contact with a spermatozoon from another male, oval-headed spermatozoa released their acrosomal enzymes (which include proteases) thereby killing rival spermatozoa.

Baker \& Bellis's (1995) kamikaze sperm hypothesis has received a great deal of uncritical media attention even though their results leave a large number of questions unanswered, particularly regarding their methods (see also Harcourt 1991; Birkhead et al. 1997; Gomendio et al. 1998; Short 1998). For example, they stated that they were apparently able to distinguish normal and acrosome-reacted human spermatozoa readily without the use of specific supravital stains or fluorescent dyes (Kohn et al. 1997), yet no other student of human spermatozoa has been able to achieve this. Indeed, most researchers agree that definitive identification of the acrosomal status of a human spermatozoon requires a specific label and that light microscopy alone (bright field or phase contrast) is unreliable (De Jonge et al. 1989). Baker \& Bellis (1995) did not label spermatozoa from different males nor did they assess either sperm viability or morphology in an objective or quantitative manner.

The aim of the present study was to investigate the kamikaze sperm hypothesis using standard andrology protocols and quantitative assessment by computerassisted sperm analysis (CASA) to address the question of whether spermatozoa from one human ejaculate can specifically influence the spermatozoa from the ejaculate of another male in vitro. Specifically, we tested the following predictions of the kamikaze sperm hypothesis: compared with self-sperm mixtures, non-self mixtures of sperm show a reduction in sperm velocity and the proportion of morphologically normal sperm and an increase in agglutination, mortality and the incidence of capacitated and acrosome-reacted sperm.

\section{MATERIAL AND METHODS}

\section{(a) Semen samples}

Ejaculates were obtained by masturbation following three days sexual abstinence from 15 donors of proven fertility attending the Jessop Hospital for Women, Sheffield, who had been screened regularly for sexually transmitted disease. The samples were processed within $1 \mathrm{~h}$ of collection. All samples used were in the normal range for sperm concentration, motility and morphology as determined by standard protocols (World Health Organization 1992): sperm concentration $46 \pm 23 \times 10^{6} \mathrm{ml}^{-1}$, sperm motility $54 \pm 18 \%$ progressively motile sperm (average path velocity $44 \pm 12 \mu \mathrm{m} \mathrm{s}^{-1}$ ) and sperm morphology $36 \pm 14 \%$ normal morphology (World Health Organization (1992) criteria) and 19 \pm \% normal morphology (strict criteria; Kruger et al. 1995). The World Health Organization (1992) criteria for normal morphology comprise sperm head length $4-5.5 \mu \mathrm{m}$, head width $2.5-3.5 \mu \mathrm{m}$, acrosome $60-70 \%$ head area and no neck, midpiece or tail defect.

All tests were done blind by one of the authors (M.M.) with no prior knowledge of the hypothesis or its predictions. CASA was used to measure the sperm motility parameters objectively as previously reported (Mohammad et al. 1996). Sperm morphology was determined using a Hobson sperm morphology analyser (Sense and Vision Ltd, Sheffield, UK) with sperm smears which had been fixed in methanol $(100 \%)$ and stained by the Papanicolaou staining procedure (World Health Organization 1992). Sperm mixtures were examined after 1 and $3 \mathrm{~h}$ incubation as this is the time that sperm normally remain in the human vagina (Tredway et al. 1978; Insler et al. 1980) and, hence, the most likely duration of sperm mixing following copulations with different males. Baker \& Bellis (1995, p. 273) provided few details of their experimental protocol, but apparently examined sperm mixtures after $3-6 \mathrm{~h}$. We considered that, with our more sensitive assays, any effects should have been apparent after the more biologically realistic interval of $3 \mathrm{~h}$.

Semen from either the same male or pairs of males was mixed and duplicate aliquots of each mixture were prepared with the order of mixing changed (i.e. $\mathrm{A}+\mathrm{B}$ and $\mathrm{B}+\mathrm{A}$ ). For each order of mixing we used paired $t$-tests to check that mixing order had no effect and we used repeatability values (Lessells \& Boag 1987) to assess the similarity of the two samples. In 12 out of 14 comparisons, the values from the different mixing orders did not differ significantly and seven out of the 14 measures of repeatabilty were significant. It is important to note that the non-significant repeatability values were obtained, as expected, from the swim-up samples (seven out of eight samples; see below) since the sperm used in these tests were a more uniform, selected sample from the whole ejaculate. Since in the majority of cases mixing order had no effect on the values obtained, we used the means from the two mixes in subsequent analyses.

\section{(b) Tests with diluted semen}

To test the most basic prediction of the kamikaze sperm hypothesis, that mixtures of semen from different men (non-self mixtures) resulted in greater aggregation of sperm than samples mixed from the same man (self mixtures), we conducted the following experiments. Ejaculates from ten different donors were mixed both with themselves and pairwise with all other donors. Aliquots $(100 \mu \mathrm{l})$ of the two semen samples were mixed together in a microcentrifuge tube diluted in $200 \mu \mathrm{l}$ of modified Earle's medium (MEM) and incubated for 1 or $3 \mathrm{~h}$ at $37^{\circ} \mathrm{C}$ in $5 \% \mathrm{CO}_{2}$ in air.

A sperm smear was made for each mixture after 1 and $3 \mathrm{~h}$ incubation. No adjustment was made for sperm concentration $\left(22-140 \times 10^{6}\right.$ sperm $\left.\mathrm{ml}^{-1}\right)$. After incubation, the sperm suspension was gently agitated by a single finger flick of the tube and a $7 \mu \mathrm{l}$ aliquot was pipetted onto a microscope slide and a cover-slip 
carefully placed on top. Cell aggregation was assessed at $\times 200$ magnification by phase-contrast microscopy. Ten fields were selected at random and the number of fields containing an aggregation of two or more spermatozoa counted after 1 and $3 \mathrm{~h}$. Aggregation was considered to have occurred when more than two fields contained two or more spermatozoa bound to each other. Sperm motility (200 sperm tracks, $1.5-3.0$ s duration per track) was assessed by CASA after 1 and $3 \mathrm{~h}$ incubation. Sperm morphology was determined for non-aggregated sperm only since it was not possible to assess the morphology of aggregated sperm either subjectively or by CASA.

\section{(c) Tests with swim-up sperm suspensions}

In order to simulate a situation resembling that when sperm have left the vagina and are 'selected' on the basis of their motility and morphology (Overstreet \& Katz 1990; Mortimer 1995), we mixed sperm, as in $\S 2(\mathrm{~b})$, in suspensions prepared from aliquots $(0.5 \mathrm{ml})$ of the same ejaculates by the swim-up method. In this, progressively motile sperm are tested after they migrate out of seminal plasma into an overlying layer of MEM (World Health Organization 1992) and are adjusted to a concentration of $10^{6}$ sperm ml ${ }^{-1}$ in MEM. The sperm aggregation test was performed as described above. In addition, a sperm viability test was performed, which measures the percentage of viable sperm, on the mixture of sperm suspensions after 1 and $3 \mathrm{~h}$ incubation using the 'Fertilyte' cell viability test (Molecular Probes Inc., Oregon, USA) as reported elsewhere (Mohammad et al. 1996).

\section{(d) Tests with fuorochrome-labelled sperm}

In order to assess whether aggregations were random with respect to the donor or, as the kamikaze sperm hypothesis predicts, more likely to comprise sperm from different males, we conducted the following experiment. A separate batch of five donors was used to prepare sperm suspensions labelled with fluorochrome. This was effective in distinguishing the origin of the spermatozoa and did not adversely affect sperm motility over the $3 \mathrm{~h}$ incubation period as assessed by CASA. Swim-up sperm suspensions were prepared as described previously and samples adjusted with MEM medium to $10^{6}$ sperm $\mathrm{ml}^{-1}$. Aliquots $(500 \mu \mathrm{l})$ were labelled with either fluorescein isothiocyanate (FITG) or tetramethyl rhodamine isothiocyanate (TRITG) as described by Lui et al. (1989) and incubated in combinations for 1 or $3 \mathrm{~h}$. A fluorochrome-labelled aliquot $(100 \mathrm{ml})$ was mixed with either a non-labelled or a differently labelled aliquot. There was no evidence that labelling had any effect on the incidence of sperm aggregations in mixtures comprising (i) FITG-labelled and TRITG-labelled sperm, (ii) FITC-labelled and unlabelled sperm, and (iii) TRITGlabelled and unlabelled sperm $\left(\chi^{2}=4.18\right.$, d.f. $=2$ and $\left.p>0.05\right)$. Labelled sperm suspensions were kept away from light (which can adversely affect cell viability) by wrapping the tubes in aluminium foil. Sperm motility and morphology was assessed after 1 and $3 \mathrm{~h}$ incubation as described above. Aggregation of spermatozoa was observed under phase-contrast and epifluorescent microscopy (Olympus (UK) Ltd) with appropriate filters for FITG $(498 \mathrm{~nm})$ and TRITC $(525 \mathrm{~nm})$ fluorescence.

\section{(e) Induction of capacitation and spontaneous acrosome reaction}

To test the hypothesis that non-self mixtures of semen resulted in a greater incidence of capacitated and acrosome-reacted sperm than self mixtures, we conducted the following experiment. The same swim-up preparations of donor ejaculates as in
$\S 2$ (c) were used to assess the phosphorylation of sperm proteins and, hence, the capacitation of sperm (Emiliozzi \& Fenichel 1997; Aitken et al. 1998; Brewis et al. 1998). Aliquots (0.5 ml) from different donors were mixed together in combinations and incubated for 1 or $3 \mathrm{~h}$ at $37^{\circ} \mathrm{C}$ in $5 \% \mathrm{CO}_{2}$ in air in capacitating MEM containing human serum albumin as described elsewhere (Brewis et al. 1996). At the end of the incubation, $100 \mu$ l aliquots of sperm suspension were removed, washed by gentle centrifugation at $600 \mathrm{~g}$ for $5 \mathrm{~min}$, resuspended in phosphate-buffered saline and each smeared onto several clean microscope slides for assessment of the sperm acrosome reaction using a monoclonal antibody (mAb 18.6) probe (Moore et al. 1987). The remainder of each aliquot was centrifuged at $5000 \mathrm{~g}$ for $5 \mathrm{~min}$ and the supernatant removed. The sperm pellet was immediately resuspended in sodium-dodecacyl sulphate sample buffer and protein analysed for phosphotyrosine residues by gel electrophoresis and immunoblotting using specific monoclonal antibody and chemiluminescent detection. The spontaneous sperm acrosome reaction was measured as a defined end-point of capacitation (Burks et al. 1995; Moore 1995; Brewis et al. 1996). The intensity of specific phosphoprotein bands, which is a measure of the extent of acrosome activation, was determined as pixel points using image analysis software (NIH Image, 1.52).

Unless stated otherwise, chemicals were from Sigma Chemical Co., Poole, Dorset, UK. Means and s.d.s are expressed. All statistical tests are two-tailed.

\section{RESULTS}

\section{(a) Tests with diluted semen}

The results of mixing combinations of ejaculates from ten donors are summarized in figure la. Non-self semen samples were no more likely to show any aggregation $(31 \%)$ than self samples $(50 \%$; see table 1$)$. There was no significant difference in the velocity of sperm after 1 or $3 \mathrm{~h}$ incubation in self and non-self mixtures (table 1 ). There was a significantly lower proportion of morphologically normal sperm in self mixtures $(30 \%)$ than in nonself mixtures $(35 \%)$ after $1 \mathrm{~h}$, although after $3 \mathrm{~h}$ the difference was not significant (table 1).

\section{(b) Tests with swim-up sperm suspensions}

The results of mixing combinations of swim-up sperm suspensions from the same ten donors are summarized in figure $1 b$. The swim-up procedure significantly reduced the incidence of aggregation (ten cases of aggregation out of 100 possible cases) compared with the diluted semen (see above) (26 cases out of $100, \chi^{2}=8.6$, d.f. $=1$ and $p<0.02$; compare figure $1 a, b)$. As expected, the sperm in swim-up suspensions were an improved subset compared with those in diluted semen (see above). For example, after $1 \mathrm{~h}$ of incubation virtually all (99-100\%) sperm in the swim-up suspension were viable. There was no evidence that swim-up suspensions from different males were any more likely to show aggregation than self mixtures (table 1). Nor were there any significant differences in the proportion of morphologically normal sperm and the velocity or viability of sperm in self and non-self mixtures after either 1 or $3 \mathrm{~h}$ incubation (table 1 ).

\section{(c) Tests with fluorochrome-labelled sperm}

There was no difference in the incidence of aggregation between self and non-self sperm labelled with fluorochrome, 
(a)

\begin{tabular}{|c|c|c|c|c|c|c|c|c|c|c|}
\hline donor & A & B & C & D & E & F & G & H & I & J \\
\hline A & X & & & & & & & & X & \\
\hline B & & X & & & & & X & & & \\
\hline C & $\mathrm{X}$ & $\mathrm{X}$ & & & & $\mathrm{X}$ & & & & \\
\hline D & & & & $\mathrm{X}$ & & & & & & $\mathrm{X}$ \\
\hline E & $\mathrm{X}$ & $\mathrm{X}$ & & & & $\mathrm{X}$ & & & $\mathrm{X}$ & \\
\hline F & & & & & $\mathrm{X}$ & & & & & \\
\hline G & & $\mathrm{X}$ & & & & & $\mathrm{X}$ & $\mathrm{X}$ & & $\mathrm{X}$ \\
\hline $\mathrm{H}$ & & & $\mathrm{X}$ & & & & $\mathrm{X}$ & & & \\
\hline $\mathrm{I}$ & & & & & $\mathrm{X}$ & & & & & $\mathrm{X}$ \\
\hline $\mathrm{J}$ & & & & $\mathrm{X}$ & & & $\mathrm{X}$ & & $\mathrm{X}$ & $\mathrm{X}$ \\
\hline
\end{tabular}

(b)

\begin{tabular}{|c|c|c|c|c|c|c|c|c|c|c|}
\hline donor & A & B & C & D & E & F & G & H & I & J \\
\hline A & & & & & & & & & & \\
\hline B & & X & & & & & & & & \\
\hline C & & & & & & & & & & \\
\hline D & & & & X & & X & & & X & \\
\hline E & X & & & & & & & & & \\
\hline F & & & & X & & & X & & & \\
\hline G & & & & & & & & & & \\
\hline H & & & & & & & & & & \\
\hline I & & & & & & & & & & \\
\hline J & & & & & & X & & X & & X \\
\hline
\end{tabular}

(c)

\begin{tabular}{|c|c|c|c|c|c|}
\hline donor & $\mathrm{R}$ & $\mathrm{S}$ & $\mathrm{T}$ & $\mathrm{U}$ & $\mathrm{V}$ \\
\hline $\mathrm{R}$ & & & $\mathrm{X}$ & & \\
\hline $\mathrm{S}$ & & & $\mathrm{X}$ & $\mathrm{X}$ & \\
\hline $\mathrm{T}$ & $\mathrm{X}$ & $\mathrm{X}$ & $\mathrm{X}$ & $\mathrm{X}$ & \\
\hline $\mathrm{U}$ & & & & & $\mathrm{X}$ \\
\hline $\mathrm{V}$ & $\mathrm{X}$ & & & & \\
\hline
\end{tabular}

Figure 1. Summary of the results of three experiments in which semen from different donors $(\mathrm{A}-\mathrm{J}$ and $\mathrm{R}-\mathrm{V})$ was mixed either with themselves (shaded boxes) or with another donor. Cells below the self-self diagonal represent cases where sperm were mixed $\mathrm{B}-\mathrm{A}, \mathrm{C}-\mathrm{A}$, etc. and cells above the diagonal indicate mixings $\mathrm{A}-\mathrm{B}, \mathrm{A}-\mathrm{C}$, etc. (a) Diluted semen only from ten donors $(\mathrm{A}-\mathrm{J})$. (b) Swim-up sperm using the same ten donors $(\mathrm{A}-\mathrm{J})$. (c) Swim-up sperm labelled with fluorochromes (see the text) from five different donors $(\mathrm{R}-\mathrm{V})$. A cross in a cell indicates that aggregation of sperm was recorded at either 1 or $3 \mathrm{~h}$ after mixing (see 2). although the sample size was relatively small (figure $1 c$ and table 1). Sperm suspensions from donor $\mathrm{T}$ had a tendency to aggregate with samples from other donors as well as with itself (figure 1c). There was no differences in the velocity of sperm in self and non-self mixtures after 1 or $3 \mathrm{~h}$ incubation (table 1), but there was a tendency for there to be fewer morphologically normal sperm in nonself mixtures (table 1). This result is consistent with the kamikaze sperm hypothesis, but since the same effect did not occur in either the diluted semen or the swim-up test (table 1), it provides little support for Baker \& Bellis's (1995) hypothesis.

We compared the observed proportion of sperm aggregations containing sperm from both males with those expected from a null model for sperm aggregations of sizes two, three to five and six or more sperm (the maximum clump size observed was 12 sperm). If sperm aggregate at random with respect to male then for aggregations of size two sperm the expected proportion of aggregations containing sperm from one or both males is predicted to be $25 \%$ that both are male A, 25\% that both are male B and $50 \%$ are mixed. For larger clumps binomial probabilities provide the predicted proportions of the various possible mixtures. As clump size increases the proportion of clumps containing sperm from both males increases, for example $87.5 \%$ for clumps of four sperm and $96.9 \%$ for clumps of six sperm. The observed proportion of sperm clumps containing sperm from both males for aggregations of two sperm did not differ significantly $\left(\chi^{2}\right.$-test $\left.p>0.05\right)$ from chance (table 2), but for larger aggregations there were significantly fewer clumps containing sperm from both males than expected by chance $\left(\chi^{2}\right.$-tests $\left.p<0.01\right)$. This suggests that sperm from the same male were significantly more likely to form aggregations.

\section{(d) Induction of capacitation and spontaneous acrosome reaction}

There was no evidence that non-self sperm mixtures resulted in any greater incidence of capacitated or acrosome-reacted sperm than self mixtures. In terms of capacitation, a three-way ANOVA (with male pair, treatment (self or non-self mixture) and time (1 or $3 \mathrm{~h}$ ) as factors) revealed that neither the effect of male pair nor treatment was significant, but, as expected, there was a significant increase in capacitation (i.e. phosphotyrosine residues in sperm proteins) with time $(p<0.001)$. The results are summarized in figure $2 a$. The same samples were also analysed in the same way for the spontaneous acrosome reaction using $\mathrm{mAb}$ 18.6. There was no effect of treatment (self versus non-self $p=0.83$ ), but there was a significant (and expected) effect of time $(p<0.001$; figure $2 a)$. In addition, there was also a significant interaction between time and male pair $(p<0.001)$, suggesting a difference between individuals in the rate at which their sperm underwent the acrosome reaction (figure $2 b$ ).

\section{DISCUSSION}

On the basis of several different in vitro assays our results provide no support for Baker \& Bellis's (1995) hypothesis that human sperm selectively make contact with sperm from a different male and then incapacitate them. 
Table 1. Summary of results of three types of test involving self-self and non-self mixtures of semen from ten different donors

(The values are means and s.d.s. The tests are either $\chi^{2}$-tests, Mann-Whitney $U$-tests $(z$-statistic) or Fisher exact tests. The units are as follows: velocity $\left(\mu \mathrm{m} \mathrm{s}^{-1}\right)$, morphology (percentage of normal head morphology) and viability (percentage viable).)

\begin{tabular}{|c|c|c|c|c|}
\hline test & self & non-self & statistic & $p$ \\
\hline \multicolumn{5}{|l|}{ diluted semen } \\
\hline incidence of aggregation & $50 \%(5 / 10)$ & $31 \%(14 / 45)$ & $\chi^{2}=1.29$ & 0.25 \\
\hline velocity $(1 \mathrm{~h})$ & $38.2 \pm 1.9$ & $39.3 \pm 0.6$ & $z=0.35$ & 0.73 \\
\hline velocity $(3 \mathrm{~h})$ & $30.7 \pm 2.5$ & $33.2 \pm 0.8$ & $z=0.88$ & 0.37 \\
\hline morphology (1 h) & $30.1 \pm 2.1$ & $35.2 \pm 0.9$ & $z=2.16$ & 0.03 \\
\hline morphology $(3 \mathrm{~h})$ & $31.6 \pm 1.9$ & $31.0 \pm 0.6$ & $z=0.17$ & 0.86 \\
\hline \multicolumn{5}{|l|}{ swim-up test } \\
\hline incidence of aggregation & $30 \%(3 / 10)$ & $13 \%(6 / 45)$ & $\chi^{2}=3.18$ & 0.02 \\
\hline velocity $(1 \mathrm{~h})$ & $52.2 \pm 1.5$ & $51.8 \pm 0.6$ & $z=0.16$ & 0.87 \\
\hline velocity $(3 \mathrm{~h})$ & $46.1 \pm 1.3$ & $45.0 \pm 0.6$ & $z=0.84$ & 0.40 \\
\hline morphology $(1 \mathrm{~h})$ & $41.9 \pm 2.5$ & $38.7 \pm 0.6$ & $\mathrm{z}=1.23$ & 0.22 \\
\hline morphology (3 h) & $40.1 \pm 1.8$ & $39.4 \pm 0.6$ & $z=0.18$ & 0.85 \\
\hline viability $(1 \mathrm{~h})$ & $99-100$ & $99-100$ & no test & - \\
\hline viability $(3 \mathrm{~h})$ & $95.5 \pm 0.9$ & $94.3 \pm 0.4$ & $z=1.03$ & 0.29 \\
\hline \multicolumn{5}{|l|}{ fluororochrome test } \\
\hline incidence of aggregation & $20 \%(1 / 5)$ & $60 \%(6 / 10)$ & Fisher exact & 0.20 \\
\hline velocity $(1 \mathrm{~h})$ & $60.6 \pm 1.7$ & $56.3 \pm 1.7$ & $z=1.46$ & 0.14 \\
\hline velocity $(3 \mathrm{~h})$ & $53.6 \pm 2.8$ & $51.0 \pm 1.2$ & $z=0.67$ & 0.50 \\
\hline morphology (1 h) & $52.8 \pm 3.1$ & $47.3 \pm 1.1$ & $z=1.90$ & 0.06 \\
\hline morphology (3 h) & $51.8 \pm 1.7$ & $46.6 \pm 1.2$ & $z=2.21$ & 0.04 \\
\hline
\end{tabular}

Table 2. Number (and percentage) of sperm aggregations containing sperm of two males in non-self mixtures with different combinations of labels in swim-up preparations from five donors

(The label combinations were green (FITG) plus red (TRITC) $(\mathrm{g}+\mathrm{r})$, green plus unlabelled $(\mathrm{g}+\mathrm{u})$ and red plus unlabelled sperm $(r+u)$ (see the text).)

number (percentage) of sperm aggregations with sperm from two males in different labelling combinations

\begin{tabular}{lccc}
$\begin{array}{l}\text { aggregation } \\
\text { size }\end{array}$ & $\mathrm{g}+\mathrm{r}$ & $\mathrm{g}+\mathrm{u}$ & $\mathrm{r}+\mathrm{u}$ \\
\cline { 2 - 4 } & $37 / 64(50)$ & $34 / 70(49)$ & $35 / 84(42)$ \\
two & $23 / 34(68)$ & $27 / 40(68)$ & $31 / 45(69)$ \\
three-five & $18 / 27(67)$ & $22 / 25(88)$ & $22 / 31(71)$ \\
six or more & $18 / 25$ \\
\hline
\end{tabular}

In several of our experiments some aggregation of spermatozoa was observed. However, in all our experiments agitation of the samples was kept to a minimum to allow any potential interactions between cells to occur. In the clinical laboratory, sperm clumping or agglutination of a few sperm in undiluted semen is common (World Health Organization 1992) and normally an ejaculate is agitated by vortexing before analysis (World Health Organization 1992). Indeed, for all the samples in the present study, agitation of an aliquot by vortexing eliminated sperm clumps indicating that the sperm interactions observed during the incubation period were weak. Within the female tract, smooth muscle action may cause considerable agitation of spermatozoa so that only strong agglutination may be maintained. Aggregation of sperm in an ejaculate may be due to incomplete liquefaction, leaving groups of spermatozoa in microscopic seminal clots. This may explain our observation that aggregations tended to be more common in self mixtures. Sperm may also adhere unselectively to cellular debris, prostatic droplets and other insoluble material in seminal plasma (World Health Organization 1992). Many previous studies have also shown that autoantibodies can be present in seminal plasma and differentially affect sperm aggregation from different ejaculates whether from the same or a different male (Shulman 1995; Sigman et al. 1997). This is probably due to sperm-coating antibodies being present in variable amounts in an ejaculate. At low titres (and occasionally at high titres), autoantibodies in seminal plasma may cause sperm aggregation without any influence on the overall fertilizing capacity of an ejaculate (Turek 1997), although individual sperm in aggregations may be less likely to participate in fertilization. Seminal plasma may also be directly detrimental to spermatozoa in contact with it for a prolonged period due to the presence of reactive oxygen molecules (Sigman et al. 1997) and over the $3 \mathrm{~h}$ incubation period in the present study we recorded a reduction in sperm velocity in several combinations of ejaculates. However, we found no difference in velocity in self and non-self mixtures of sperm.

Mixing undiluted semen from different ejaculates in vitro to some extent mimics sequential copulation with different partners over a short period of time when ejaculates could intermingle within the vagina before any selection processes occur in the female tract. This situation might arise in primates such as the chimpanzee Pan troglodytes where sperm competition is intense, copulation frequency is high and females are routinely inseminated by several males and often in rapid succession (e.g. Gagneux et al. 1997, 1999). In humans sperm competition is less intense (Smith 1984b; Harcourt et al. 1995 Gomendio et al. 1998), female copulation frequency lower 

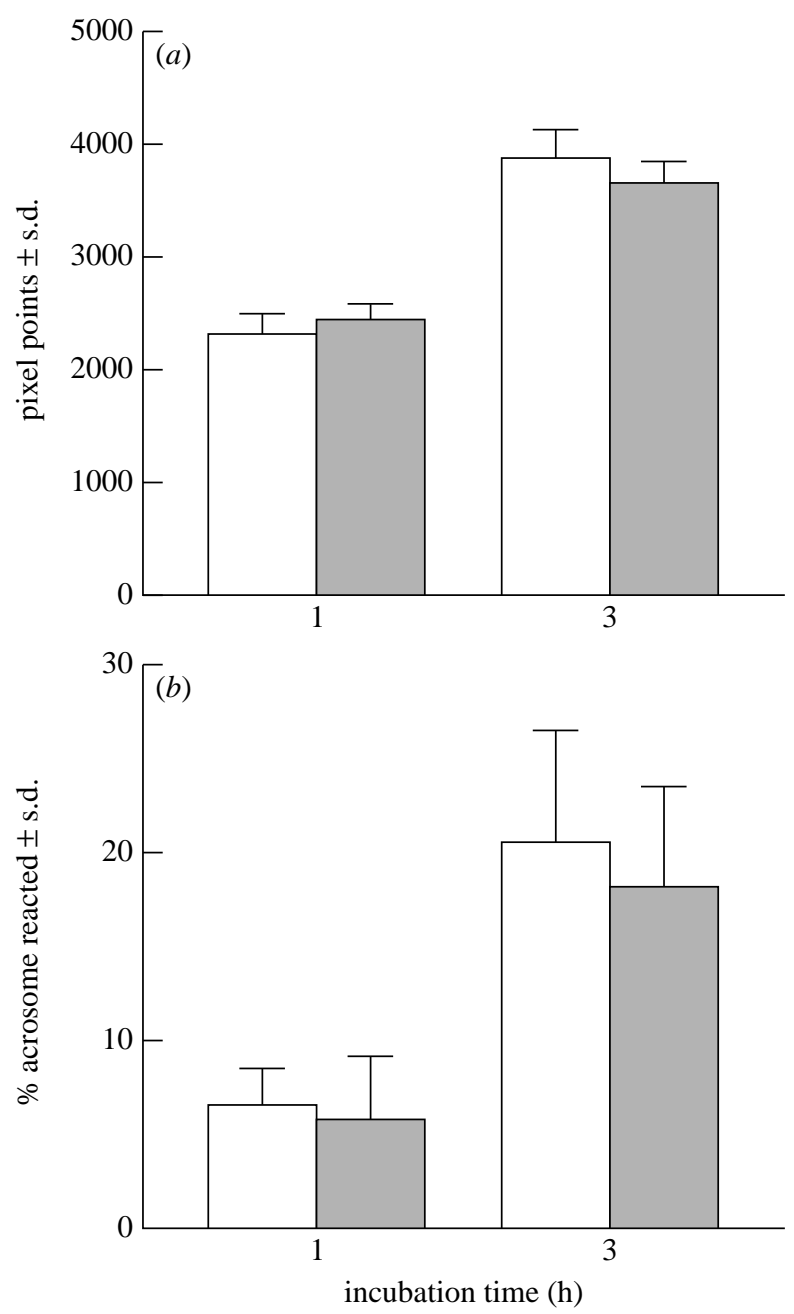

Figure 2. (a) Phosphotyrosine expression of the $95 \mathrm{kDa}$ epitope as determined by image analysis (mean \pm s.d. pixel points) for self (unshaded) and non-self (shaded) mixtures of sperm. This assay provides an index of the incidence of capacitation (see the text). The difference with incubation time was significant (ANOVA $p<0.001$ ), but there was no difference between self and non-self mixtures. $(b)$ The difference with incubation time was significant (ANOVA $p<0.001$ ), but there was no difference between self and nonself mixtures of semen.

and the interval between successive inseminations likely to be longer. As a result it is less likely that undiluted semen samples and more likely that selected sperm from different ejaculates would encounter each other in the female tract. Within a few hours of insemination, spermatozoa involved in fertilization pass into reservoirs in the cervical mucus and enter the uterine lumen and the isthmus region of the oviduct (Overstreet \& Katz 1990; Mortimer 1995). Spermatozoa in these reserves are probably selected on the basis of their progressive motility and morphology characteristics and occur at much lower concentrations $\left(\times 10^{-3}\right.$ to $\left.10^{-6}\right)$ than those deposited initially in the vagina at coitus. There is therefore much less opportunity for direct sperm-sperm contact once spermatozoa leave the vagina. In vitro swim-up sperm preparations represent such selected cells since this method allows spermatozoa with good progressive motility and possibly more normal morphology (World Health Organization 1992) to swim from the remainder of the ejaculate (Yovich 1995). Although we found a significant improvement in the mean sperm morphology after the swim-up procedure, spermatozoa with an ovalhead morphology, which Baker \& Bellis (1995) indicated were involved in 'warfare', were not excluded by this procedure. Nonetheless, mixing experiments involving equal numbers of swim-up spermatozoa from different donors provided no evidence in support of selective interactions between spermatozoa from the ejaculates of different men.

One of the major criticisms of the study by Baker \& Bellis (1995) is the lack of validation of their results. It is impossible to determine whether sperm agglutinations are due to self or non-self interactions or a combination of both on the basis of light microscopy alone. By fluorescent labelling of spermatozoa of one or both ejaculates the composition of a clump was determined unequivocally. The mixing experiments with labelled spermatozoa indicated that, in clumps comprising two sperm, self and non-self clumps were equally likely to occur but, with larger clumps, self aggregations were more likely than non-self aggregations. It could be argued that this result is consistent with the kamikaze sperm hypothesis if, as Baker \& Bellis (1995, p. 268) proposed, self sperm aggregate to form a block to subsequent inseminations. However, had we found that sperm from different males were more likely to form aggregations, that could also have been construed as evidence that sperm from one male had blocked the progress of the other. Consequently, at best this result provide equivocal support for the kamikaze sperm hypothesis.

If a kamikaze spermatozoon acts by undergoing a spontaneous acrosome reaction in the vicinity of a foreign spermatozoon then one would expect non-self sperm mixtures to show higher rates of capacitation and a higher proportion of acrosome-reacted spermatozoa compared with self mixtures (Baker \& Bellis 1995). A number of previous reports have shown that the amount of tyrosine-phosphorylated epitopes in human spermatozoa is related to their capacitation status (Brewis \& Moore 1997). Our results clearly indicated that tyrosine phosphorylation increased during incubation but that this was not related to the mixture of sperm samples. Similarly, the proportion of sperm that had undergone the acrosome reaction did not differ between self and non-self mixtures.

Baker \& Bellis (1995) used additional circumstantial evidence from the literature to support their kamikaze sperm hypothesis, including the phenomenon of differential fertilizing capacity. When semen pooled from several different males is used for artificial insemination (as in breeding in many domestic animals) one male usually fertilizes a disproportionate number of offspring (Dziuk 1996). Baker \& Bellis (1995, p. 302) suggested that the differential fertilizing capacity 'will only make sense when viewed in the perspective of the KSH' (kamikaze sperm hypothesis). However, recent studies on nonhumans, while not explicitly testing the kamikaze sperm hypothesis, have shown that the differential fertilizing capacity can be adequately explained by inherent differences in the attributes of the sperm of different males (Birkhead et al. 1999; Donoghue et al. 1999).

In summary, there is no evidence from this study to support the conclusions reached by Baker \& Bellis (1995) 
that particular spermatozoa of an ejaculate act in a 'kamikaze' manner against spermatozoa of a different ejaculate. In a mixture of ejaculates, there may be interactions between spermatozoa but in vitro most of these interactions appear to be random and do not involve specific recognition processes.

We are grateful to the staff of the Andrology Laboratory, Jessop Hospital for Women, for organization of the donor semen samples used in this study. We also wish to thank Professor J. D. Biggins for statistical advice and Dr B. J. Hatchwell, Dr F. M. Hunterand, Dr E. R. S. Roldan and four anonymous referees for their constructive comments on the manuscript.

\section{REFERENCES}

Aitken, R. J., Harkiss, D., Knox, W., Paterson, M. \& Irvine, D. S 1998 A novel signal transduction cascade in capacitating human spermatozoa characterised by a redox-regulated CAMP-mediated induction of tyrosine phosphorylation. $\mathcal{F}$. Cell Sci. 111, 645-656.

Baker, R. R. \& Bellis, M. A. 1988 Kamikaze sperm in mammals? Anim. Behav. 36, 936-939.

Baker, R. R. \& Bellis, M. A. 1989 Elaboration of the kamikase sperm hypothesis: a reply to Harcourt. Anim. Behav. 37, 865-867.

Baker, R. R. \& Bellis, M. A. 1995 Human sperm competition. London. Chapman \& Hall.

Birkhead, T. R. 1998 Sperm competition in birds. Rev. Reprod. 3, 123-129.

Birkhead, T. R. \& Møller, A. P. 1998 Sperm competition and sexual selection. London. Academic Press.

Birkhead, T. R. \& Parker, G. A. 1997 Sperm competition and mating systems. In Behavioural ecology: an evolutionary approach (ed. J. R. Krebs \& N. B. Davies), pp. 121-145. Oxford, UK: Blackwell.

Birkhead, T. R., Moore, H. D. M. \& Bedford, J. M. 1997 Sex, science and sensationalism. Trends Ecol. Evol. 12, 121-122.

Birkhead, T. R., Martinez, J. G., Burke, T. \& Froman, D. P. 1999 Sperm mobility determines the outcome of sperm competition in the domestic fowl. Proc. R. Soc. Lond. B 266, 1-6.

Brewis, I. \& Moore, H. D. M. 1997 Molecular mechanisms of gamete recognition and fusion at fertilization. Hum. Reprod. 12(Suppl. 2), 156-165.

Brewis, I. A., Clayton, R., Barratt, C. L. R., Hornby, D. P. J \& Moore, H. D. M. 1996 Recombinant human zona pellucida glycoprotein 3 induces calcium influx and acrosome reaction in human spermatozoa. Mol. Hum. Reprod. 2, 583-589.

Brewis, I. A., Clayton, R., Browes, C. E., Martin, H., Barratt, C. L. R., Hornby, D. P. \& Moore, H. D. M. 1998 Tyrosine phosphorylation of a $95 \mathrm{kDa}$ protein and induction of the acrosome reaction in human spermatozoa by recombinant human zona pellucida glycoprotein 3. Mol. Hum. Reprod. 12, $1136-1144$

Burks, D. J., Carballada, R., Moore, H. D. M. \& Saling, P. M. 1995 A novel tyrosine kinase from human sperm interacts with the zona pellucida at fertilization. Science 269, 83-86.

Chapman, T., Liddle, L. F., Kalb, J. M., Wolfner, M. F. \& Partridge, L. 1995 Cost of mating in Drosophila melanogaster females is mediated by male accessory gland products. Nature 373, 241-244.

Clark, A. G., Aguade, M., Prout, T., Harshman, L. G. \& Langley, C. H. 1995 Variation in sperm displacement and its association with accessory gland protein in Drosophila melanogaster. Genetics 139, 189-201.

Clark, A. G., Begun, D. J. \& Prout, T. 1999 Female $\times$ male interactions in Drosophila sperm competition. Science 283, 217-220.

Cohen, J. 1967 Correlation between chiasma frequency and sperm redundancy. Nature 215, 862-863.
Cohen, J. 1973 Crossovers, sperm redundancy, and their close associations. Heredity 31, 408-415.

De Jonge, C. J., Mack, S. R. \& Zaneveld, L. J. D. 1989 Synchronous assay for human sperm capacitation and acrosome reaction. f. Androl. 10, 232-239.

Donoghue, A. M., Sonstegard, T. S., King, L. M., Smith, E. J. \& Burt, D. W. 1999 Turkey sperm mobility influences paternity in the context of competitive fertilization. Biol. Reprod. 61, 422-427.

Dzuik, P. J. 1996 Factors that influence the proportion of offspring sired by a male following heterospermic insemination. Anim. Reprod. Sci. 43, 65-88.

Emiliozzi, C. \& Fenichel, P. 1997 Protein tyrosine phosphorylation is associated with capacitation of human sperm in vitro but is not sufficient for its completion. Biol. Reprod. 56, 674-679.

Froman, D. P. \& Feltman, A. J. 1998 Sperm mobility: a quantitative trait in the domestic fowl (Gallus domesticus). Biol. Reprod. 58, 379-384.

Gagneux, P., Woodruff, D. S. \& Boesch, C. 1997 Furtive mating in female chimpanzees. Nature 387, 358-359.

Gagneux, P., Boesch, C. \& Woodruff, D. S. 1999 Female reproductive strategies, paternity and community structure in wild West African chimpanzees. Anim. Behav. 57, 19-32.

Ginsberg, J. R. \& Huck, U. W. 1989 Sperm competition in mammals. Trends Ecol. Evol. 4, 74-79.

Gomendio, M., Harcourt, A. H. \& Roldan, E. R. S. 1998 Sperm competition in mammals. In Sperm competition and sexual selection (ed. T. R. Birkhead \& A. P. Møller). London: Academic Press.

Harcourt, A. H. 1991 Sperm competition and the evolution of nonfertilizing sperm in mammals. Evolution 45, 314-328.

Harcourt, A. H., Purvis, A. \& Liles, L. 1995 Sperm competition: mating system, not breeding season, affects testes size of primates. Funct. Ecol. 9, 468-476.

Harshman, L. G. \& Prout, T. 1994 Sperm displacement without sperm transfer in Drosophila melanogaster. Evolution 48, 758-766.

Holt, C., Holt, W. V., Moore, H. D. M., Reed, H. C. B. \& Curnock, R. M. 1996 Objectively measured boar sperm motility parameters correlate with the outcome of on-farm inseminations. Results of two fertility trials. F. Androl. 18, 312-323.

Huck, U. W., Tonias, B. A. \& Lisk, R. D. 1989 The effectiveness of competitive male inseminations in golden hamsters, Mesocricetus auratus, depends on an interaction of mating order, time delay between males and the time of mating relative to ovulation. Anim. Behav. 37, 674-680.

Insler, V., Glezerman, M., Ziedel, L., Bernstein, D. \& Misgar, N. 1980 Sperm storage in the human cervix: a quantitative study. Fertil. Steril. 33, 288-293.

Kohn, F.-M., Mack, S. R., Schill, W.-B. \& Zaneveld, L. J. D. 1997 Detection of human sperm acrosome: comparison between methods using double staining, Pisum sativum agglutinin, concanavalin A and transmission electron microscopy. Hum. Reprod. 12, 714-721.

Kruger, T. F., Dutoit, T. C., Franken, D. R., Menkveld, R. \& Lombard, C. J. 1995 Strict morphology-assessing the agreement between the manual method (strict criteria) and the sperm morphology analyzer IVOS. Fertil. Steril. 63, 134-141.

Lessells, C. M. \& Boag, P. T. 1987 Unrepeatable repeatabilities: a common mistake. Auk 104, 116-121.

Lui, D. Y., Clarke, G. N., Lopata, A., Johnston, W. I. H. \& Baker, H. W. G. 1989 A sperm-zona pellucida binding test and in vitro fertilization. Fertil. Steril. 52, 281-287.

Manning, J. T. \& Chamberlain, A. T. 1994 Sib-competition and sperm competiveness: an answer to 'Why so many sperms?' and the recombination/sperm number correlation. Proc. $R$. Soc. Lond. B 256, 177-182. 
Mohammad, S. N., Barratt, C. L., Cooke, I. D. \& Moore, H. D. M. 1996 Continuous assessment of human spermatozoa viability during cryopreservation. F. Androl. 18, 43-50.

Moore, H. D. M. 1995 Modification of sperm membrane antigens during capacitation. In Human acrosome reaction (ed. P. Fenichel \& J. Parinaud), pp. 35-43. Paris: John Libbey.

Moore, H. D. M \& Bedford, J. M. 1983 The interaction of mammalian gametes in the female. Sperm/egg interactions in vivo. In Mechanism and control of animal fertilization (ed. J. F. Hartmann), pp. 453-497. New York: Academic Press.

Moore, H. D. M., Hartman, T. D. \& Smith, C. A. 1987 A monoclonal antibody for marking the human acrosome reaction. Gamete Res. 17, 245-259.

Mortimer, D. 1995 Sperm transport in the female tract. In Gametes: the spermatozoon (ed. J.G. Grudzinsksa \& J. Yovich), pp. 157-174. Cambridge University Press.

Overstreet, J. W. \& Katz, D. F. 1990 Interaction between the female reproductive tract and spermatozoa. In Controls of sperm motility: biological and clinical aspects (ed. C. Gagnon), pp. 63-75. Boca Raton, FL: CRC Press.

Parker, G. A. 1970 Sperm competition and its evolutionary consequences in the insects. Biol. Rev. 45, 525-567.

Price, C. S. C., Dyer, K. A. \& Coyne, J. A. 1999 Sperm competition between Drosophila males involves both displacement and incapacitation. Nature 400, 449-452.

Short, R. V. 1998 Review of human sperm competition: copulation, masturbation and infidelity by R. R. Baker and M. A. Bellis. Eur. Sociobiol. Soc. Nereslett. 47, 20-23.

Shulman, S. 1995 Immunological reactions and infertility. In Immunobiology of human reproduction (ed. M. Kurpisz \& N. Fernandez), pp. 53-78. Oxford, UK: Bios Scientific Publishers Ltd.

Sigman, M., Lipshultz, L. I. \& Howards, S. S. 1997 Evaluation of the subfertile male. In Infertility in the male (ed. L. I.
Lipshultz \& S. S. Howards), pp. 173-193. St Louis, MO: Mosby-Year Book Inc.

Silberglied, R. E., Shepherd, J. G. \& Dickinson, J. L. 1984 Eunuchs: the role of apyrene sperm in Lepidoptera? Am. Nat. 123, 255-265.

Simmons, L. W., Parker, G. A. \& Stockley, P. 1999 Sperm displacement in the yellow dungfly, Scatophaga stercoraria: an investigation of male and female processes. Am. Nat. 153, 302-314.

Sivinski, J. 1980 Sexual selection and insect sperm. Fl. Entomol. 63, 99-111.

Smith, R. L. 1984a Sperm competition and the evolution of animal mating systems. Orlando, FL: Academic Press.

Smith, R. L. $1984 b$ Human sperm competition. In Sperm competition and the evolution of animal mating systems (ed. R. L. Smith), pp. 601-659. Orlando, FL: Academic Press.

Tredway, D. R., Buchanan, G. C. \& Drake, T. S. 1978 Comparison of the fractional postcoital test and semen analysis. Am. 7. Obstet. Gynecol. 130, 647-652.

Turek, P. J. 1997 Immunopathology and infertility. In Infertility in the male (ed. L. I. Lipshultz \& S. S. Howards), pp. 305-325. St Louis, MO: Mosby-Year Book Inc.

World Health Organization 1992 Laboratory manual for the examination of human semen and sperm-cervical mucus interactions. Cambridge University Press.

Yovich, J. L. 1995 Sperm preparation for assisted conception. In Gametes: the spermatozoon (ed. J. G. Grudzinsksa \& J. Yovich), pp. 268-281. Cambridge University Press.

Zeh, J. A. \& Zeh, D. W. 1997 The evolution of polyandry. II. Post-copulatory defences against genetic incompatibility. Proc. R. Soc. Lond. B 264, 69-75.

As this paper exceeds the maximum length normally permitted, the authors have agreed to contribute to production costs. 